\title{
Volutaria tubuliflora (Murb.) Sennen (Asteraceae), nueva especie alóctona asilvestrada para Chile
}

\section{Volutaria tubuliflora (Murb.) Sennen (Asteraceae), a new alien species in Chile}

\author{
Sebastián Telllier ${ }^{1 *}$, Jorge Macaya ${ }^{2}$, Alfonso Susanna ${ }^{3} \&$ Juan Antonio Calleja ${ }^{4}$ \\ ${ }^{1}$ Escuela de Arquitectura del Paisaje, Universidad Central de Santiago de Chile. Santa Isabel 1186, Santiago, Chile. \\ ${ }^{2}$ Consultor independiente. Los Nogales 823, Providencia, Santiago, Chile. \\ ${ }^{3}$ Botánica, Dpto. de Biología Animal, Vegetal y Ecología, Universidad Autónoma de Barcelona, ES-08193 Bellaterra, España. \\ ${ }^{4}$ Instituto Botánico de Barcelona (IBB-CSIC-ICUB), pg. del Migdia, s/n, ES-08038 Barcelona, España. \\ *steillier@gmail.com
}

\begin{abstract}
Volutaria tubuliflora (Asteraceae-Carduae) is described as a new alien plant species in Chilean vascular flora. The species has the widest range of natural geographical distribution of the genus being present from southern Europe to Arabic peninsula, inclusive Macaronesia. In Chile it has been naturalized in the Desert ecoregion, and it growths in the Atacama Region (III). Plants were found at Panamericana highway border, and apparently still have not invaded outside. In this paper we provide morphological and ecological information for this species.
\end{abstract}

Volutaria Cassini pertenece a las Asteraceae (Compuestas), tribu Carduae, subtribu Centaureinae. De acuerdo con Susanna \& Garcia-Jacas (2007), es uno de los géneros basales de la subtribu y está constituido por una veintena de especies.

La distribución de la mayor parte de las especies se circunscribe a dos polos de diversidad, uno localizado en el noroeste de África y Macaronesia y otro en el entorno del golfo de Adén (Etiopía, Somalia, Yemen, Arabia Saudí y Socotra). Algunas, como $V$. bollei, $V$. djibutensis o $V$. socotrensis, son microendemismos restringidos a islas o sistemas montañosos de reducida extensión; otras, por el contrario, tienen áreas de distribución muy amplias. Entre estas últimas destaca sin duda $V$. tubuliflora, que aparece en todo el norte de África, desde Marruecos hasta Egipto; también en el sur de Europa, en España, Sicilia y Turquía; en la Macaronesia, en todas las Canarias e incluso en la Península Arábiga, con lo que su área de distribución geográfica resulta la más amplia de todo el género, y su expansión podría ser muy reciente (Calleja, en prep.).

Se trata de una planta bien adaptada al clima de tipo mediterráneo árido y a su transición con el clima desértico. Coloniza ambientes nitrificados de origen antrópico, donde es muy frecuente a lo largo de todo tipo de vías, tales como pistas, carreteras y autopistas, y en cultivos de secano y de regadío (Calleja, obs. pers., Sánchez Gullón et al. 2006, Devesa \& López en prensa). En este último caso aparece citada en terrenos desérticos de Arabia Saudí (Calleja, en prep.).

Volutaria tubuliflora se comporta como una especie oportunista cuyas semillas germinan apenas les son favorables las condiciones de humedad. Su forma de crecimiento corresponde al de una hierba anual, pero ocasionalmente puede tener un ciclo vital más dilatado.

Las plantas tienen una talla muy variable, desde los 15 $\mathrm{cm}$ hasta superar ampliamente el metro de altura. Cuanto mayor es la humedad más altura alcanza, desarrolla hojas más grandes y más numerosas, y produce un mayor número de inflorescencias (Calleja, obs. pers.); la morfología de los capítulos, de las flores y de los frutos es, sin embargo, menos heterogénea (Devesa \& López en prensa), aunque existen poblaciones que poseen capítulos con flores periféricas bien desarrolladas y numerosas, y otras cuyos capítulos carecen de ellas (Murbeck 1897, 1923, Pitard 1913). El color de las flores periféricas y de las centrales se describe invariablemente como púrpura o violáceo; sin embargo, en áreas muy áridas del suroeste de Marruecos existen poblaciones con capítulos con pocas flores periféricas y éstas de color blanco (Calleja, obs. pers.).

Hasta hoy no existían registros de Volutaria tubuliflora ni para Chile ni para Sudamérica en general (Marticorena y Quezada 1985; bases de datos Cono Sur y Trópicos).

En relación con su hallazgo en Chile, se reporta una población de esta especie que se ha naturalizado a lo largo 
de la carretera Panamericana entre las ciudades de Copiapó y Vallenar, particularmente entre la quebrada Algarrobal y las afueras de Vallenar. El área señalada es conocida en la fitoecología de Chile como parte de la Región del Desierto, subregión del Desierto Florido, en la formación "Desierto Florido de los Llanos" (Gajardo, 1998), caracterizada por precipitaciones de frecuencia y cuantía muy variable entre años, con episodios de sequía pluvial de hasta diez años. Las lluvias, escasas, están asociadas a los años denominados como "Niño" (Rundel et al. 1991, Armesto et al. 1993, Dillon \& Hoffmann 1997), que gatillan la floración masiva de arbustos y suculentas, así como la emergencia de las hierbas perennes y las anuales. Dado su aislamiento geográfico, es una zona que presenta un alto endemismo, sobre un $50 \%$ de acuerdo con Letelier et al. (2008). Complementariamente, Luebert \& Pliscoff (2006) indican que el área corresponde al piso de vegetación del matorral desértico mediterráneo interior de Skytanthus acutus Meyen (Apocynaceae) y Atriplex deserticola Phil. (Chenopodiaceae), que se extiende entre los 200 y los $1500 \mathrm{~m}$ de altitud, principalmente en la Región de Atacama (III). De acuerdo con los mismos autores, este piso de vegetación ocupa el piso de bioclima mesomediterráneo ultrahiperárido.

\section{DESCRIPCIÓN}

Volutaria tubuliflora (Murb.) Sennen, Campagn. Bot. Maroc. Or. 1930-5, 148. 1936.

Basiónimo: Amberboa tubuliflora Murb., Act. Univ. Lund. 33(2): 105. 1897.

Amberboa lippii DC. subsp. tubuliflora Murb. in Contrib. Fl. Moroc. II, Lunds Univ. Arsskr., 12: 1-126. 1923.

Hierba anual, con pelos araneosos y glandulíferos. Tallos de $25-60 \mathrm{~cm}$ de altura, ramificados desde la parte media. Hojas presentes durante la antesis, glandulosas, las basales sésiles, rosuladas (Fig. 1A), las del medio y las superiores alternas, también sésiles y decurrentes; hojas basales con lámina lirado-pinnatipartida, de hasta $90 \mathrm{~mm}$ de longitud, con 3-5 lóbulos aserrado-mucronados, mucrón apical cónico y amarillento; las superiores hasta pinnatisectas disminuyendo en tamaño hacia el ápice y estrechísimas. Inflorescencias dispuestas en corimbos terminales con 3-5 cabezuelas en cada ramificación, pedúnculo principal $30-40 \mathrm{~cm}$ de longitud, en la parte ramificada, cada capítulo es portado por un pedúnculo de $1-5 \mathrm{~cm}$ de longitud; involucro de $c a$. $10 \mathrm{~mm}$ de ancho x $15 \mathrm{~mm}$ de altura, brácteas numerosas, dispuestas en 4-5 filas (Fig. 1B), dorso largamente hirsuto, con tres nervaduras bien marcadas; las externas con el ápice fuertemente mucronado, de color más oscuro, de 6-8 $\mathrm{mm}$ de longitud, las internas, algo más largas, sin mucrón, pero con el ápice membranoso. Flores solamente de color blanco; de $6 \mathrm{~mm}$ de longitud con el tubo densamente velloso por fuera, lóbulos muy breves. Aquenios oblongo-ovoides, con costillas con puntuaciones y pelos muy largos y densos; vilano de escamas, hialino-blanquecinas, más o menos obovado-lineares, de 2-4 mm de longitud (Fig. 1C).

\section{Material estudiado}

Región de Atacama: provincia del Huasco, carretera Panamericana, entre la quebrada Algarrobal y el cruce a Vallenar, km 670 (28¹3’50 S;7041’'18 W), 9-10-2013, S. Teillier \& J. Delaunoy 7875 (CONC).

La población crece en el margen de la carretera, donde se beneficia de las escasas precipitaciones y de la condensación de la neblina frecuente en el lugar; por ahora, al parecer por la casi ausencia de precipitaciones en 2013, no se la observó en sitios aledaños (Fig. 1D). Se han observado y fotografiado ejemplares de la especie, en el valle del río Elqui, ubicado a unos $300 \mathrm{~km}$ al SE del sitio de recolección (René Bustamante, com. pers.).

Resulta notable y particular la presencia de ejemplares sólo con flores blancas, que en su área natural son escasos.

Es posible que esta cita sea la primera para América del Sur. Respecto de su procedencia, resulta complejo plantear una hipótesis. En el área no existen puertos agrícolas que faciliten la llegada y el establecimiento desde los países donde está documentada su presencia. Tampoco se la ha sindicado como alóctona asilvestrada o como cultivada en algún país vecino. En su lugar de origen la especie está en un proceso de expansión, tal como lo atestigua su presencia en irrigaciones instaladas en el siglo XX en Arabia Saudita, su aparición en el SO de la península Ibérica (Sánchez Gullón et al. 2006) y, muy especialmente, este hallazgo en Chile. Estas citas coinciden, a su vez, con la dispersión reciente de la especie en su área de distribución natural inferida a partir de datos moleculares (Calleja, en prep.). En Chile, es posible que los primeros ejemplares se establecieran en el valle del Huasco — donde está la ciudad de Vallenar-, sitio que presenta mejores condiciones hídricas comparadas con las condiciones climáticas zonales. Dada su versatilidad para colonizar ambientes alterados, es seguro que su llegada a América ha sido reciente, producto de una dispersión mediada por el ser humano, como el transporte de especies forrajeras, o quizá con el uso de maquinaria de obras públicas ya utilizada en Europa. A partir de estas circunstancias, el viento o el ganado doméstico y silvestre pueden estar dispersando la especie hacia distintos enclaves con clima árido de Chile, hasta ahora en los llanos de la depresión intermedia de la Región de Atacama y en el valle del Elqui, Región de Coquimbo. 


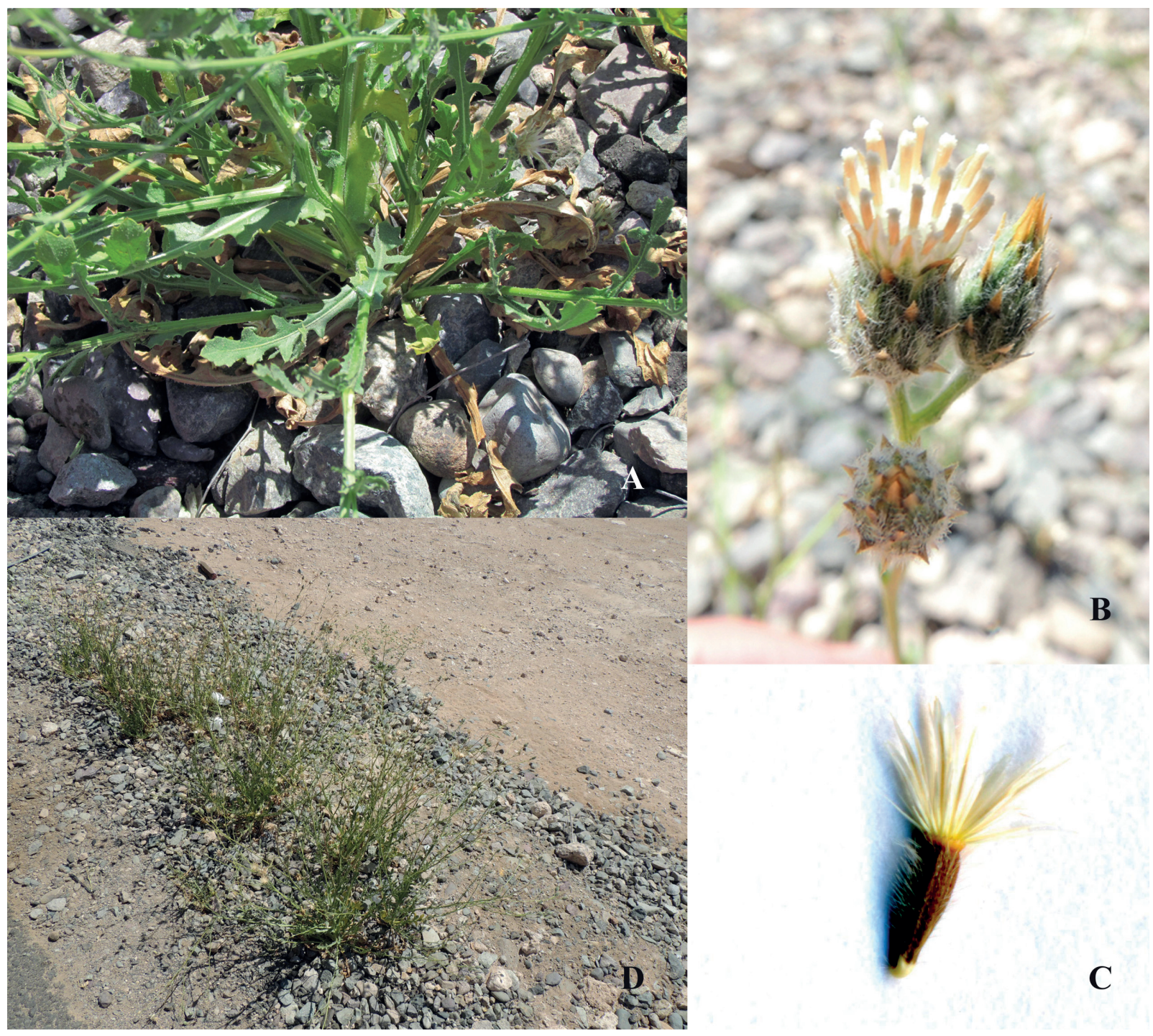

Figure 1. Volutaria tubuliflora A. Hojas basales. B. Capítulos y flores (Fotografía Javiera Delaunoy). C. Aquenio, tamaño real, 3 mm de longitud sin vilano. D. Ubicación de las plantas en el hábitat.

Figura 1. Volutaria tubuliflora A. Basal leafs. B. Flowers and capitula (Photograph by Javiera Delaunoy). C. Achene, real size, 3 mm without pappus. D. Plants growing at their habitat.

\section{AGRADECIMIENTOS}

Los autores agradecen a Javiera Delaunoy, Andrea Zapata y a la empresa SGA-Consultores por la ayuda en terreno.

\section{BIBLIOGRAFÍA}

Armesto, J.J., P.E. Vidiella \& J.R. Gutiérrez. 1993. Plant communities of the fog-free coastal desert of Chile: plant strategies in a fluctuating environment. Revista Chilena de Historia Natural 66: 271-282.

Devesa, J.A. \& J. López. (en prensa). Volutaria Vaill. En: S.
Castroviejo, M. Laínz, G. López-González, P. Montserrat, F. Muñoz-Garmendia, J. Paiva \& L. Villar (eds.), Flora Ibérica Vol. 16. CSIC, Madrid.

Dillon, M. \& A.E. Hoffmann 1997. Lomas formations of the Atacama desert, Northern Chile. In: S.D. Davis, V.H. Heywood, O. Herrera-McBryde, J. Villa-Lobos \& A.C. Hamilton (eds.), Centres of plant diversity: a guide and strategy for their conservation, pp. 528-535. WWF Information Press, Oxford.

Gajardo, R. 1998. La vegetación natural de Chile. Editorial Universitaria, Santiago de Chile. 165 pp.

Letelier, L., F.A. Squeo, G. Arancio, A. Marticorena, M. MuñozSchick, M.T.K. Arroyo, P. León-Lobos, S. Montecinos \& J.R. Gutiérrez. 2008. Diversidad vegetal de la Región 
de Atacama, Chile. En: F.A. Squeo, G. Arancio, J.R. Gutiérrez, L. Letelier, M.T.K. Arroyo, P. León-Lobos \& L. Rentería-Arrieta (eds.), Flora Incluida en categoría de conservación de la Región de Atacama y estrategias para su conservación. Ediciones Universidad de La Serena, La Serena. viii $+72 \mathrm{pp}$.

Luebert, F. \& P. Pliscoff. 2006. Sinopsis bioclimática y vegetacional de Chile. Editorial Universitaria, Santiago de Chile. 316 pp.

Marticorena, C. \& M. Quezada. 1985. Catálogo de la flora vascular de Chile. Gayana Botánica (Chile) 42(1-2): 1-157.

Murbeck, S. 1897. Contributions à la connaissance de la flore du nord-ouest de l'Afrique; et plus spécialment de la Tunisie. E. Malmström, Lund. 83 pp.

MurBeCK, S. 1923. Contributions à la connaissance de la Flore du Maroc, II. Acta Universitatis Lundensis, Nova series, 12: 1-126.

Pitard, C.-J. 1913. Exploration scientifique du Maroc I. Masson, Paris. 187 pp.
Rundel, P.W., M.O. Dillon, B. Palma, H.A. Mooney, S.L. Gulmon \& J.R. Ehleringer. 1991. The phytogeography and ecology of the coastal Atacama and Peruvian desert. Aliso 13(1): 1-49.

Sánchez Gullón, E., F.J. Macías Fuentes \& P. Weickert. 2006. Algunas especies adventicias o naturalizadas en la provincia de Huelva (SO de España). Lagascalia 26: 180187.

Susanna, A. \& N. Garcia-Jacas. 2009. Cardueae (Carduoideae), In: V.A. Funk, A. Susanna, T.F. Stuessy \& R.J. Bayer (eds.), Systematics, evolution and biogeography of Compositae, pp. 293-313. IAPT, Viena.

TROPICOS.ORG. Missouri Botanical Garden. 22 Apr 2014 $<$ http://www.tropicos.org > :http://www.tropicos.org/home Consultada en diciembre de 2013.

Zuloaga, F.O., O. Morrone \& M.J. Belgrano. 2008. Catálogo de las plantas vasculares del cono sur (Argentina, Sur de Brasil, Chile, Paraguay y Uruguay). http://www2.darwin. edu.ar/Proyectos/FloraArgentina/FA.asp. Consultada en diciembre de 2013.

Recibido: 11.12 .13

Aceptado: 23.04.14 